\title{
On the Eigenvalue Based Detection for Multiantenna Cognitive Radio System
}

\author{
Syed Sajjad Ali, ${ }^{1}$ Chang Liu, ${ }^{1}$ Jialong Liu, ${ }^{1}$ Minglu Jin, ${ }^{1}$ and Jae Moung Kim ${ }^{2}$ \\ ${ }^{1}$ School of Information and Communication Engineering, Dalian University of Technology, Dalian 116024, China \\ ${ }^{2}$ Department of Information and Communication Engineering, INHA University, Incheon 402-751, Republic of Korea \\ Correspondence should be addressed to Jae Moung Kim; jaekim@inha.ac.kr
}

Received 29 December 2015; Revised 4 April 2016; Accepted 5 May 2016

Academic Editor: Yunfei Chen

Copyright (c) 2016 Syed Sajjad Ali et al. This is an open access article distributed under the Creative Commons Attribution License, which permits unrestricted use, distribution, and reproduction in any medium, provided the original work is properly cited.

\begin{abstract}
Eigenvalue based spectrum sensing can make detection by catching correlation features in space and time domains, which can not only reduce the effect of noise uncertainty, but also achieve high detection probability. Hence, the eigenvalue based detection is always a hot topic in spectrum sensing area. However, most existing algorithms only consider part of eigenvalues rather than all the eigenvalues, which does not make full use of correlation of eigenvalues. Motivated by this, this paper focuses on multiantenna system and makes all the eigenvalues weighted for detection. Through the analysis of system model, we transfer the eigenvalue weighting issue to an optimal problem and derive the theoretical expression of detection threshold and probability of false alarm and obtain the close form expression of optimal solution. Finally, we propose new weighting schemes to give promotions of the detection performance. Simulations verify the efficiency of the proposed algorithms.
\end{abstract}

\section{Introduction}

The rapid development of wireless services leads to the scarcity of the public radio spectrum becoming more and more serious. Traditionally, licensed spectrum is allocated over relatively long time periods and is intended to be used only by legitimate users. Cognitive radio (CR) technology was proposed to handle the contradiction between the shortage of spectrum resource and the underutilization of licensed spectrum $[1,2]$. Spectrum sensing which is a fundamental task of $\mathrm{CR}$ is aimed at obtaining the awareness of licensed spectrum usage and existence of primary users (PUs) in a specific geographical location [3-7]. The main function of spectrum sensing is to frequently explore the spectrum holes for the secondary users (SUs) by detecting the presence of primary users so that the SUs can share the licensed spectrum. Therefore, spectrum sensing becomes critical in cognitive radio system.

There have been many discussions and proposed solutions for spectrum sensing [8]. Of these methods, likelihood ratio test (LRT) [9], cyclostationary detection (CSD) [10, 11], and matched filtering (MF) detection $[12,13]$ can achieve optimal performance while requiring both source signal and noise power information, which is not available in practice. Hence, semiblind methods such as energy detection (ED) $[9,14]$ and maximum eigenvalue detection (MED) [15] are proposed. Among these, ED is the most commonly chosen scheme for study and implementation due to its relatively low complexity and satisfactory performance under low signalto-noise ratio (SNR) environment. However, ED heavily relies on the accuracy of the knowledge of noise power which is generally changing over time. This so-called noise uncertainty problem [16] can significantly degrade the performance of ED algorithm.

To overcome these shortcomings, blind detection algorithms which require no information on source signal or noise power have been intensively studied recently [17-21]. The classical blind detection algorithms are the eigenvalue based methods. For example, maximum-minimum eigenvalue (MME) detection [22], arithmetic to geometric mean (AGM) detection [23], and signal-subspace eigenvalues (SSE) method [23] can overcome the shortcoming of ED and 
achieve outstanding performance. On the other hand, eigenvalue based methods have also been studied in new scenarios, such as cooperative adaptive versions [24] and Multiple Primary Transmit Power (MPTP) scenario [25].

However, most algorithms only consider part of eigenvalues, such as maximum, minimum, and mean value, which does not make full use of all the eigenvalues to make detection. Motivated by this, we focus on the problem of eigenvalue weighting in multiantenna system and analyze the related problems. By analyzing the model of eigenvalue weighting, we transfer the weighting problem to an optimal problem. Using the latest random matrix theory (RMT) [26, 27], we derive the close form expression of probability of detection and probability of false alarm and obtain the optimal solution. Finally, we propose new weighting schemes to give promotions of the detection performance. Simulations verify the efficiency of the proposed algorithms. The main contributions of this paper include the following:

(i) Different from the traditional eigenvalue based detection, we consider making detection by utilizing all of the eigenvalues in the multiantenna system. By transferring the weighting problem to an optimal problem, we analyze and derive an energy based maximum ratio combination (EN-MRC) method.

(ii) Considering the case of correlated signals is common in applications, we use the idea of MRC weighting in EN-MRC method to design an eigenvalue based MRC (EIG-MRC) scheme: signal eigenvalue weighting (SEW) based detection, which needs the a priori information of signals' covariance matrix and noise power.

(iii) To make the detection more practical, we use the maximum likelihood estimation (MLE) approach to design a method of signal eigenvalue approximation weighting (SEAW) based detection, in which only the noise power is needed.

The rest of the paper is organized as follows. Section 2 explains the system model. The eigenvalue weighting based detection is studied in Section 3. Section 4 presents simulation results and conclusion is presented in Section 5. Some notations used in the paper are listed as follows: superscripts $T$ and $H$ stand for transpose and Hermitian transpose (transpose-conjugate), respectively.

\section{System Model}

Figure 1 illustrates a classical multiantenna spectrum sensing scenario with $D$ randomly distributed primary users (PU in figure) and $P$ randomly distributed secondary users (SU in figure). Once PUs begin to communicate, the surrounding SUs can receive the PU signals and then capture the samples to operate the spectrum sensing.

According to Figure 1, the SUs are equipped with $M$ receiving antennas and there are $D$ PU signals arriving in the antenna array. In this case, the sensing problem in multiantenna cognitive radio system can be written as

$$
\begin{aligned}
& H_{0}: x_{i}(k)=n_{i}(k) \\
& H_{1}: x_{i}(k)=\sum_{j=1}^{D} h_{i j} s_{j}(k)+n_{i}(k),
\end{aligned}
$$

where $i=1,2, \ldots, M$ represents the $i$ th receiving antenna and $k=0,1, \ldots, N-1$ is the $k$ th sample. $x_{i}(k)$ is the sample of the $i$ th receiving antenna. $h_{i j}$ is the channel gain between the $j$ th PU signal $s_{j}(k)$ and the $i$ th receiving antenna. $n_{i}(k)$ is the additive white Gaussian noise (AWGN) with 0 mean, $\sigma_{n}^{2}$ variance.

Stacking the samples at the same time we can get the following receiving vector of antenna array:

$$
\begin{aligned}
\mathbf{x}(k) & =\left[x_{1}(k), x_{2}(k), \ldots, x_{M}(k)\right]^{T}, \\
\mathbf{s}(k) & =\left[s_{1}(k), s_{2}(k), \ldots, s_{D}(k)\right]^{T}, \\
\mathbf{n}(k) & =\left[n_{1}(k), n_{2}(k), \ldots, n_{M}(k)\right]^{T} .
\end{aligned}
$$

Hence, formula (1) can be rewritten as the matrix form:

$$
\begin{aligned}
& H_{0}: \mathbf{X}=\mathbf{N}, \\
& H_{1}: \mathbf{X}=\mathbf{H S}+\mathbf{N},
\end{aligned}
$$

where $\mathbf{X}=[\mathbf{x}(0), \mathbf{x}(1), \ldots, \mathbf{x}(N-1)]^{T}$ and $\mathbf{N}=[\mathbf{n}(0), \mathbf{n}(1)$, $\ldots, \mathbf{n}(N-1)]^{T}$ are the antenna receiving matrix and noise matrix, respectively. $\mathbf{H} \in \mathbb{C}^{M \times D}$ is the channel gain matrix of the signal matrix $\mathbf{S}=[\mathbf{s}(0), \mathbf{s}(1), \ldots, \mathbf{s}(N-1)]^{T}$.

\section{Eigenvalue Weighting Based Detection}

3.1. Fundamental of Eigenvalue Weighting Based Detection. Based on (3), the corresponding covariance matrix can be written as

$$
\begin{aligned}
& \mathbf{R}_{\mathbf{X}}=E\left(\mathbf{X X}^{H}\right), \\
& \mathbf{R}_{\mathbf{S}}=E\left(\mathbf{S S}^{H}\right), \\
& \mathbf{R}_{\mathbf{N}}=E\left(\mathbf{N N}^{H}\right) .
\end{aligned}
$$

Hence, we can rewrite $\mathbf{R}_{\mathbf{X}}$ as

$$
\mathbf{R}_{\mathbf{X}}=\mathbf{H R}_{\mathbf{S}} \mathbf{H}^{H}+\mathbf{R}_{\mathbf{N}} \cdot
$$

Let $\lambda_{1} \geq \lambda_{2} \geq \cdots \geq \lambda_{M}$ and $\rho_{1} \geq \rho_{2} \geq \cdots \geq \rho_{D}$ represent the eigenvalues of $\mathbf{R}_{\mathbf{X}}$ and $\mathbf{H R}_{\mathbf{S}} \mathbf{H}^{H}$, respectively. Obviously, when PUs are present, we can get $\lambda_{i}=\rho_{i}+\sigma_{n}^{2}$; when PUs are absent, that is, $\mathbf{R}_{\mathbf{X}}=\mathbf{R}_{\mathbf{N}}$, we can have $\lambda_{1}=\lambda_{2}=\cdots=\lambda_{M}=\sigma_{n}^{2}$.

Based on the analysis above, we can make detection by weighting the eigenvalues. Considering the number of samples is finite in reality we can get the following test statistic:

$$
T=\sum_{i=1}^{M} w_{i} \lambda_{i}\left(\mathbf{R}_{\mathbf{X}}(N)\right),
$$




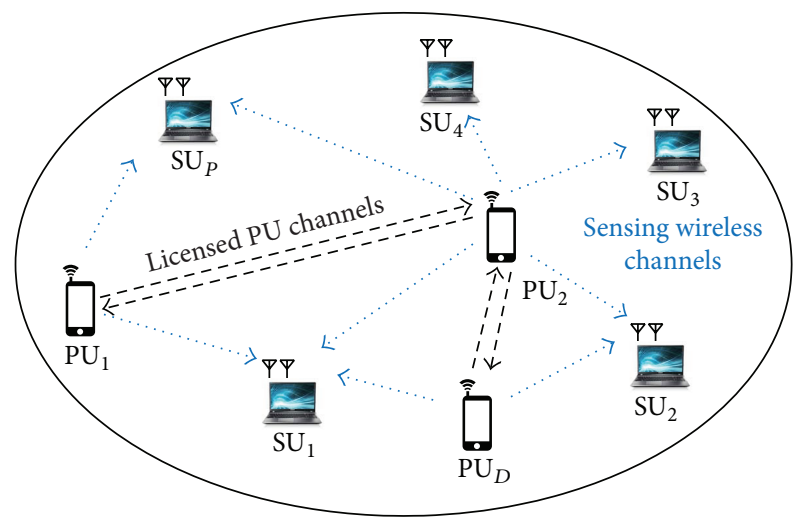

$--\rightarrow$ Licensed PU channels

$\cdots>$ Sensing wireless channels

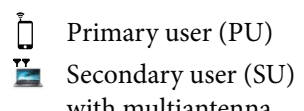

FIGURE 1: Scenario of spectrum sensing for multiantenna cognitive radio system.

where $\lambda(\cdot)$ is the eigenvalues and $w_{i}$ is the weighting coefficient. $\mathbf{R}_{\mathbf{X}}(N)=(1 / N) \mathbf{X} \mathbf{X}^{H}$ is the samples covariance matrix. Obviously, if $T>\gamma$ ( $\gamma$ is the test threshold), then PUs are present; otherwise, PUs are absent.

Finally, we summarize the general eigenvalue weighting algorithm steps as follows.

Eigenvalue Weighting Based Spectrum Sensing Algorithm for Multiantenna Cognitive Radio System

Step 1 (compute the sample covariance matrix of the received signal). Since the number of samples is finite, we can only use the sample covariance matrix $\mathbf{R}_{\mathbf{X}}(N)=(1 / N) \mathbf{X X}^{H}$.

Step 2 (obtain the eigenvalues of sample covariance matrix). Make eigenvalue decomposition (EVD) of $\mathbf{R}_{\mathbf{X}}(N)$, obtain $M$ eigenvalues, and sort them in a descending order: $\lambda_{1} \geq \lambda_{2} \geq$ $\cdots \geq \lambda_{M}$.

Step 3 (calculate the test statistic of the eigenvalue weighting). Let all the eigenvalues be weighted by $w_{i}$ and compute the sum of them. Thus, we can obtain the test statistic in (6).

Step 4 (decision). If $T>\gamma$, then signal exists ("yes" decision); otherwise, signal does not exist ("no" decision), where $\gamma$ is a threshold.

3.2. Theoretical Analysis of Eigenvalue Weighting Based Detection. Note that how to select weights $w_{i}$ is of great importance, which can affect the performance of the algorithm directly. Based on the Neyman-Pearson rule, we can express the weighting selection problem as the following optimal problem $[28,29]$ :

$$
\begin{array}{ll}
\max _{\mathbf{w}} & P_{d}=\int_{r}^{\infty} f_{T \mid H_{1}}(x ; \mathbf{w}) d x \\
\text { s.t. } & P_{f a}=\int_{r}^{\infty} f_{T \mid H_{0}}(x ; \mathbf{w}) d x,
\end{array}
$$

where $\mathbf{w}=\left[w_{1}, w_{2}, \ldots, w_{M}\right]^{T}$ is the weighting coefficient vector; $P_{d}$ and $P_{f a}$ represent the probability of detection and the probability of false alarm. $f_{T \mid H_{1}}(\cdot)$ and $f_{T \mid H_{0}}(\cdot)$ are the probability density function of test statistic under $H_{1}$ and $H_{0}$, respectively.

Based on (6), we find that it is possible to analyze the distribution of the test statistic whereas the joint probability density function is rather complex, whose close form expression is not available. However, we can transfer the problem of eigenvalue weighting of the matrix to a problem of the trace of a new matrix and the analysis of distribution of the trace is a simple problem. The detailed analysis is showed in the following.

Let $\mathbf{Y}=\mathbf{G X} \in \mathbb{C}^{M \times N}$ and $\mathbf{G}=\operatorname{diag}\left[g_{1}, g_{2}, \ldots, g_{M}\right]=$ $\operatorname{diag}\left[\sqrt{w_{1}}, \sqrt{w_{2}}, \ldots, \sqrt{w_{M}}\right]^{T}$. Hence,

$$
\mathbf{W}_{\mathbf{Y}}=\mathbf{Y} \mathbf{Y}^{H}=\mathbf{G W}_{\mathbf{X}} \mathbf{G}^{H},
$$

where $\mathbf{W}_{\mathbf{X}}=\mathbf{X} \mathbf{X}^{H}$. When the number of samples $N$ tends to infinite, the $\mathbf{W}_{\mathbf{X}}$ tends to a diagonal matrix and we can get the following:

$$
\operatorname{EVD}\left(\mathbf{W}_{\mathbf{Y}}\right)=\operatorname{EVD}\left(\mathbf{G W}_{\mathbf{X}} \mathbf{G}^{H}\right) \approx \mathbf{G E V D}\left(\mathbf{W}_{\mathbf{X}}\right) \mathbf{G}^{H},
$$

where $\operatorname{EVD}(\cdot)$ represents the diagonal matrix of eigenvalues and the equality holds when the number of samples $N$ tends to infinite. Hence, if we make eigenvalue weighting of $\mathbf{W}_{\mathbf{X}}$ by $\mathbf{w}=\left[w_{1}, w_{2}, \ldots, w_{M}\right]^{T}$ and calculate the sum of the eigenvalues after weighting, then it is equivalent to compute the trace of $\mathbf{W}_{\mathbf{Y}}$. Since $\mathbf{W}_{\mathbf{X}}=N \mathbf{R}_{\mathbf{X}}(N)$, we can rewrite (6) as the following:

$$
\begin{aligned}
T & =\sum_{i=1}^{M} w_{i} \lambda_{i}\left(\mathbf{R}_{\mathbf{X}}(N)\right) \approx \sum_{i=1}^{M} \lambda_{i}\left(\mathbf{R}_{\mathbf{Y}}(N)\right) \\
& =\operatorname{Trace}\left(\mathbf{R}_{\mathbf{Y}}(N)\right)=\operatorname{Trace}\left(\mathbf{G R}_{\mathbf{X}}(N) \mathbf{G}^{H}\right) \\
& =\frac{1}{N} \sum_{i=1}^{M} \sum_{k=0}^{N-1} w_{i}\left|r_{\mathbf{X} i}(k)\right|^{2},
\end{aligned}
$$

where $r_{\mathbf{X} i}(k)$ is the $i$ th row, $(k+1)$ th element of $\mathbf{R}_{\mathbf{X}}(N)$. Let $a_{i}=\sum_{k=0}^{N-1}\left|r_{\mathbf{X} i}(k)\right|^{2}$ and thus the test statistic $T$ can be written as

$$
T^{\prime}=N T=\sum_{i=1}^{M} w_{i} a_{i}
$$

For simplification, we assume the noise variance $\sigma_{n}^{2}=1$. When the number of samples is large enough we can get the following expression based on central limit theorem (CRT):

$a_{i}$

$$
\sim \begin{cases}\mathcal{N}\left(N \sum_{i=1}^{M} w_{i}, 2 N \sum_{i=1}^{M} w_{i}^{2}\right), & H_{0} \\ \mathcal{N}\left(N \sum_{i=1}^{M} w_{i}\left(1+r_{i}\right), 2 N \sum_{i=1}^{M} w_{i}^{2}\left(1+2 r_{i}\right)\right), & H_{1},\end{cases}
$$


where $r_{i}=E\left|\sum_{j=1}^{D} h_{i j} s_{j}(k)\right|^{2}$ is the power of the PU signals. Therefore, we can obtain the expressions of $P_{f a}$ and $P_{d}$, respectively:

$$
\begin{aligned}
P_{f a} & =P\left\{T^{\prime}>\gamma \mid H_{0}\right\}=Q\left(\frac{\gamma-N \sum_{i=1}^{M} w_{i}}{\sqrt{2 N \sum_{i=1}^{M} w_{i}^{2}}}\right), \\
P_{d} & =P\left\{T^{\prime}>\gamma \mid H_{1}\right\} \\
& =Q\left(\frac{\gamma-N \sum_{i=1}^{M} w_{i}\left(1+r_{i}\right)}{\sqrt{2 N \sum_{i=1}^{M} w_{i}^{2}\left(1+2 r_{i}\right)}}\right),
\end{aligned}
$$

where $Q(x)=\int_{x}^{+\infty}(1 / \sqrt{2 \pi}) e^{-t^{2} / 2} d t$. Hence, based on (13) and (14), we can finally get the expression as

$$
P_{d}=Q\left(\frac{Q^{-1}\left(P_{f a}\right)-\sqrt{(N / 2) \sum_{i=1}^{M} \alpha_{i} r_{i}}}{\sqrt{\sum_{i=1}^{M} \alpha_{i}^{2}\left(1+2 r_{i}\right)}}\right),
$$

where $\alpha_{i}=w_{i} / \sqrt{\sum_{i=1}^{M} w_{i}^{2}}$ and $\sum_{i=1}^{M} \alpha_{i}^{2}=1$. Since the SNR of spectrum sensing is rather low $(-20 \mathrm{~dB})$, which leads to $r_{i} \ll 1$, we can get $\sum_{i=1}^{M} \alpha_{i}^{2}\left(1+2 r_{i}\right) \approx 1$. Hence, (15) can be approximated as

$$
P_{d} \approx Q\left(Q^{-1}\left(P_{f a}\right)-\sqrt{\frac{N}{2} \sum_{i=1}^{M} \alpha_{i} r_{i}}\right) .
$$

Therefore, problem (7) can be rewritten as

$$
\begin{aligned}
\max _{\alpha} & \sum_{i=1}^{M} \alpha_{i} r_{i} \\
\text { s.t. } & \sum_{i=1}^{M} \alpha_{i}^{2}=1 .
\end{aligned}
$$

Note that this problem can be solved by Lagrangian multiplier method and the solution is written as

$$
\alpha_{i}^{*}=\frac{r_{i}}{\sqrt{\sum_{i=1}^{M} r_{i}^{2}}} .
$$

Let $\sum_{i=1}^{M} w_{i}^{2}=1$ and we can finally get the weighting coefficient:

$$
w_{i}^{*}=\alpha_{i}^{*}, \quad 1 \leq i \leq M
$$

Note that this weighting scheme is exactly identical to the maximal ratio combination (MRC) weighting scheme in [28, 29] and we call it energy based MRC (EN-MRC) detection. Hence, by studying the idea of MRC weighting scheme, we apply this idea into eigenvalue weighting and finally develop a kind of energy based MRC algorithm. The test statistic can be written as

$$
T_{\mathrm{EN}-\mathrm{MRC}}=\sum_{i=1}^{M} \frac{r_{i}}{\sqrt{\sum_{i=1}^{M} r_{i}^{2}}} \lambda_{i}
$$

where $r_{i}=E\left|\sum_{j=1}^{D} h_{i j} s_{j}(k)\right|^{2}$ is the power of the PU signals.
3.3. Eigenvalue Weighting Based Detection. Note that the transformation from eigenvalue to energy in (9) is approximately equivalent and the equality holds when $N$ tends to infinite. Hence, the corresponding analysis should be more accurate when the number of samples tends to be very large. On the other hand, the analysis under this case is based on the assumption that the received signals are independent and identically distributed (i.i.d.) for each other, which is not very accurate for the case of highly correlated signals. For example, as for (12), the distribution of $a_{i}$ under $H_{1}$ is considered as a linear combination of Gaussian variables with $\left(1+r_{i}\right)$ variance, which is based on the assumption that the received signals under $H_{1}$ are i.i.d. for each other. However, this assumption is only available under a cooperative spectrum sensing model whose samples are collected from different sensing nodes. Hence, the weighting coefficient in (20) is not an appropriate weighting scheme especially for the case of highly correlated signals and thus it needs to be improved for better catching the signals' correlation.

Motivated by this, we try to analyze the weighting coefficients from the aspect of eigenvalue directly. Since $r_{i}=$ $E\left|h_{i j} s_{j}(k)\right|^{2}$ is the power of the PU signals and $E\left[\left|h_{i j}\right|^{2}\right]=$ 1 , we can then replace the power of the PU signals $r_{i}$ in (20) with the eigenvalues of signal covariance matrix $\rho_{i}=$ $\left[\operatorname{EVD}\left(\mathbf{H R}_{\mathbf{S}} \mathbf{H}^{H}\right)\right]_{i}$.

In this case, the test statistic can further capture the correlation among signals and may achieve better performance especially when there are highly correlated PU signals. Hence, we propose a signal eigenvalue weighting (SEW) based detection and the test statistic is given as

$$
T_{\mathrm{SEW}}=\sum_{i=1}^{M} \frac{\rho_{i}}{\sqrt{\sum_{i=1}^{M} \rho_{i}^{2}}} \lambda_{i}
$$

where $\lambda_{i}$ and $\rho_{i}$ are the eigenvalues of sample and signals' covariance matrix, respectively. Although the SEW based detection may perform better performance, it is not available in practice as it needs the a priori information of the channel, signal, and noise. Hence, we try to use the maximum likelihood estimation (MLE) of these parameters to design semiblind detection, in which only noise power is needed. Hence, we will analyze and derive the MLE of eigenvalues of the PU signals' covariance matrix in the following.

According to the analysis in [23], the MLE of signals' covariance matrix $\mathbf{R}_{\mathrm{s}}$ can be expressed as

$$
\begin{aligned}
\widehat{\mathbf{R}}_{\mathbf{s}} & =\mathbf{U}_{\mathbf{x}} \operatorname{Diag}\left(\left(\lambda_{1}-\sigma_{n}^{2}\right)^{+},\left(\lambda_{2}-\sigma_{n}^{2}\right)^{+}, \ldots,\right. \\
& \left.\left(\lambda_{M}-\sigma_{n}^{2}\right)^{+}\right) \mathbf{U}_{\mathbf{x}}^{H},
\end{aligned}
$$

where $\mathbf{U}_{\mathbf{x}}$ is the eigenvector of sample covariance $\mathbf{R}_{\mathbf{x}}(N)$ and $(x)^{+}=\max (0, x)$ represents the maximum between $x$ and 0 . Hence, the MLE of eigenvalues of PU signals' covariance matrix can be written as

$$
\widehat{\rho}_{i}=\left(\lambda_{i}-\sigma_{n}^{2}\right)^{+}
$$




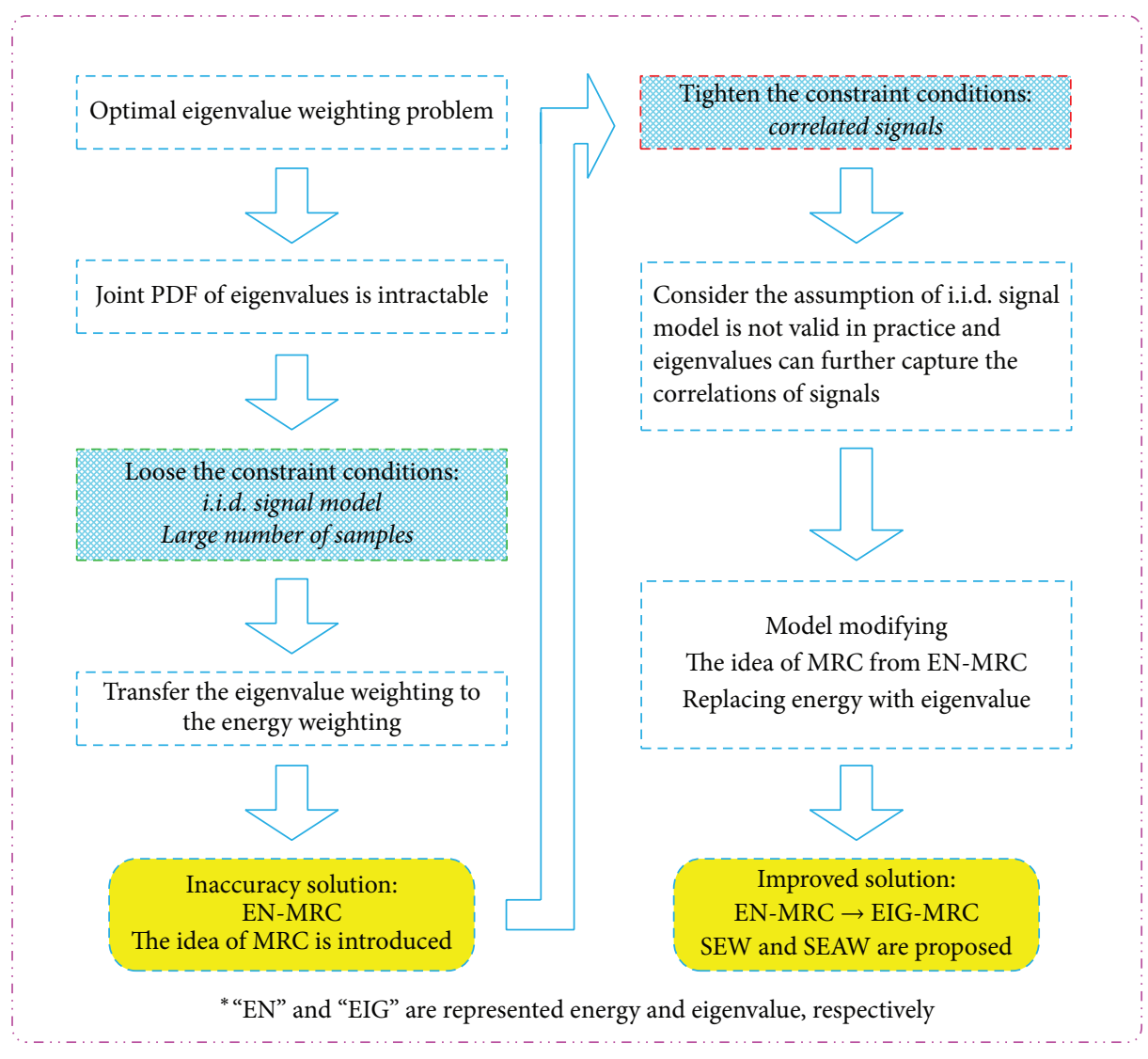

FIGURE 2: Illustration of how to obtain the eigenvalue weighting schemes.

Substituting the MLE of signal eigenvalue into (21) we can obtain the test statistic of signal eigenvalue approximation weighting (SEAW) based detection as

$$
T_{\mathrm{SEAW}}=\sum_{i=1}^{M} \frac{\left(\lambda_{i}-\sigma_{n}^{2}\right)^{+}}{\sqrt{\sum_{j=1}^{M}\left(\left(\lambda_{j}-\sigma_{n}^{2}\right)^{+}\right)^{2}}} \lambda_{i}
$$

As a summary, we propose three weighting schemes: one is traditional MRC based detection (i.e., EN-MRC) and the other two are improvement eigenvalue weighting schemes, that is, SEW based detection and SEAW based detection. For the convenience of comparison, we summarize these three methods in Table 1.

Remark. Since eigenvalue weighting problem can not be solved directly, we first loose the constraint conditions and assume that the PU signals follow the i.i.d. model and the number of samples is very large. In this case, we can obtain an inaccuracy solution: EN-MRC. Based on the MRC weighting scheme, we then tighten the constraint conditions and modify the assumption to make it satisfy the requirements of the practical system, that is, correlated signal model. Considering the eigenvalues can further capture the correlations of signals, we finally replace the energies with eigenvalues and design the eigenvalue based MRC (EIG-MRC) schemes: SEW and
SEAW based detection. The corresponding illustration is shown in Figure 2.

\section{Simulations and Discussions}

This section provides some simulation results for multiantenna cognitive radio systems in the MATLAB environment. Since this paper focuses the eigenvalue weighting schemes for spectrum sensing, we will compare the proposed EN-MRC, SEW, and SEAW based detection with eigenvalue based methods, including MED, MME, and AGM detection. We assume there is $1 \mathrm{PU}$ or $2 \mathrm{PUs}$ transmitting signal over the Nakagami- $m(m=1)$ channel in presence of AWGN. The SUs are equipped with 4-element antenna array. The stopping criterion set is at 10000 iterations and the $P_{f a}$ is set as 0.1 (this has been specified as the maximum allowable $P_{f a}$ by the WRAN 802.22 working group).

The simulation results of detection performance in terms of number of samples $N=100$ with 1 PU and 2 PUs are presented in Figures 3 and 4, respectively. It is shown that when compared with eigenvalue based methods, such as MED, MME, and AGM, the proposed SEW and SEAW based detection perform much higher probability of detection with different SNRs, while the EN-MRC performs a relatively lower detection probability when compared with MED, MME, and AGM detection. It is because algorithms SEW and SEAW are regarded as "EIG-MRC" weighting scheme and MED and 
TABle 1: Promotion schemes of eigenvalue weighting based spectrum sensing algorithm.

\begin{tabular}{|c|c|c|}
\hline Algorithm & Test statistic & Priori conditions \\
\hline $\begin{array}{l}\text { Energy based maximum ratio } \\
\text { combination (EN-MRC) }\end{array}$ & $\begin{array}{c}T_{\mathrm{EN}-\mathrm{MRC}}=\sum_{i=1}^{M} \frac{r_{i}}{\sqrt{\sum_{i=1}^{M} r_{i}^{2}}} \lambda_{i} \\
\begin{array}{c}\text { where } \lambda_{i} \text { and } r_{i} \text { are the eigenvalues of sample and } \\
\text { the power of the PU signals, respectively. }\end{array}\end{array}$ & PU signals' energy and noise power \\
\hline $\begin{array}{l}\text { Signal eigenvalue weighting } \\
\text { (SEW) based detection }\end{array}$ & $\begin{array}{c}T_{\mathrm{SEW}}=\sum_{i=1}^{M} \frac{\rho_{i}}{\sqrt{\sum_{i=1}^{M} \rho_{i}^{2}}} \lambda_{i} \\
\text { where } \lambda_{i} \text { and } \rho_{i} \text { are the eigenvalues of sample and } \\
\text { PU signals' covariance matrix, respectively. }\end{array}$ & $\begin{array}{c}\text { Eigenvalues of PU signals' covariance matrix } \\
\text { and noise power }\end{array}$ \\
\hline $\begin{array}{l}\text { Signal eigenvalue approximation } \\
\text { weighting (SEAW) based } \\
\text { detection }\end{array}$ & $\begin{array}{c}T_{\text {SEAW }}=\sum_{i=1}^{M} \frac{\left(\lambda_{i}-\sigma_{n}^{2}\right)^{+}}{\sqrt{\sum_{j=1}^{M}\left(\left(\lambda_{j}-\sigma_{n}^{2}\right)^{+}\right)^{2}}} \lambda_{i} \\
\text { where } \lambda_{i} \text { is the eigenvalues of sample covariance } \\
\text { matrix; } \sigma_{n}^{2} \text { is the noise power at receiver. }\end{array}$ & Noise power at receiver \\
\hline
\end{tabular}

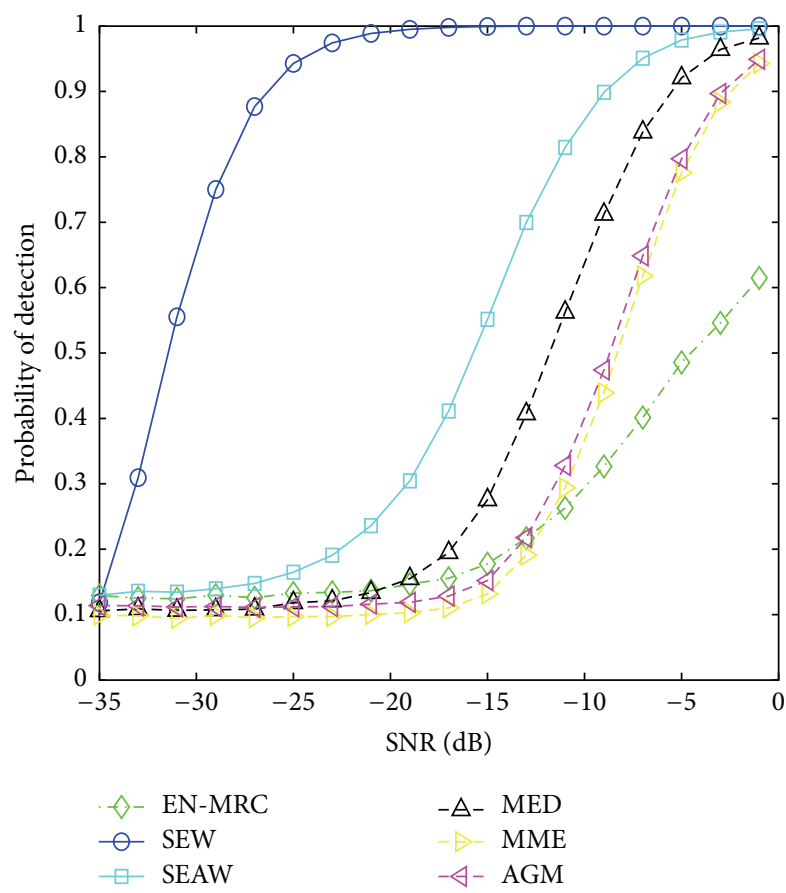

FIGURE 3: Detection performance under $N=100$ with $1 \mathrm{PU}$.

AGM belong to the selection combination (SC) and equal gain combination (EGC) weighting schemes for eigenvalues, respectively. As for EN-MRC, it is the energy based weighting coefficients, which can not fully capture the correlations. In addition, the MME is just a kind of partial eigenvalue based nonweighting detection and thus it has limited detection performance. However, since low SNR approximation has been adopted to derive the EN-MRC scheme, the EN-MRC is able to achieve a relatively higher detection probability. For example, the EN-MRC is slightly better than MME and AGM when the $\mathrm{SNR}$ is ranging from $-35 \mathrm{~dB}$ to $-13 \mathrm{~dB}$. On the other hand, when the SNR increases, the probability of detection of EN-MRC drops a little and presents a slightly worse performance (since the number of eigenvalues for the simulation

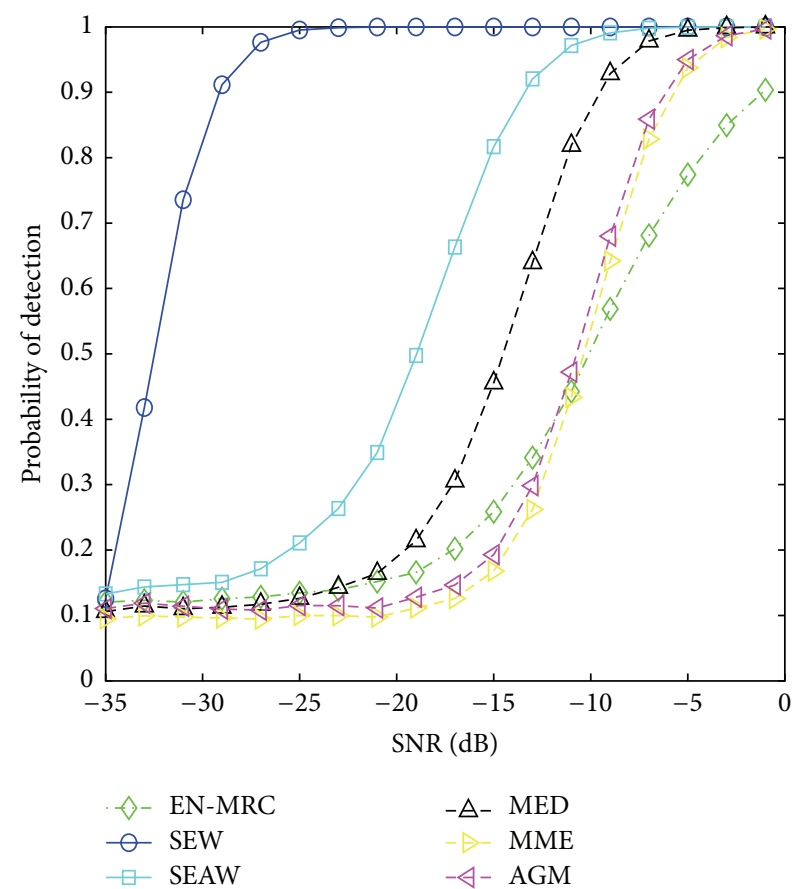

FIGURE 4: Detection performance under $N=100$ with 2 PUs.

is very small, the advantages of making detection by using all the eigenvalues or the energies are not obvious, which means the AGM or EN-MRC may not achieve a better performance than MME). When comparing Figure 3 with Figure 4, we can find that the performance increases with the increasing number of PUs, such as a nearly $30 \%$ detection probability improvement in terms of $-15 \mathrm{~dB}$.

Similarly, Figures 5 and 6 present the simulation results of probability of detection in terms of number of samples $N=1000$ with 1 PU and 2 PUs. Again, the proposed SEW and SEAW methods achieve a higher detection performance and the EN-MRC outperforms MME and AGM under low SNRs. Hence, the simulation results can further verify that it is just 


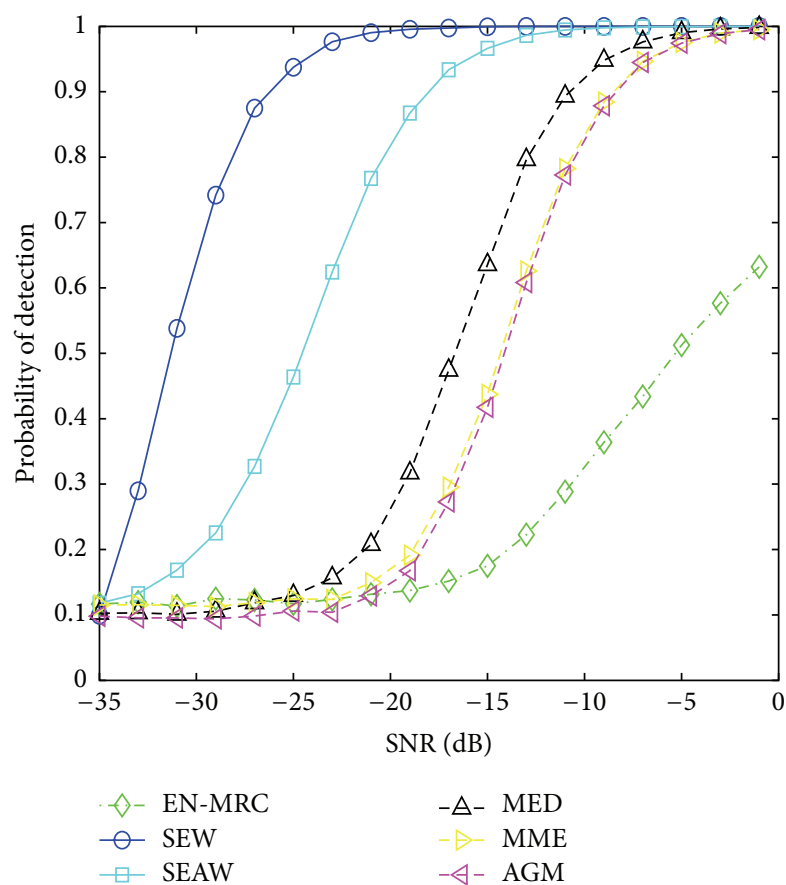

FIGURE 5: Detection performance under $N=1000$ with 1 PU.

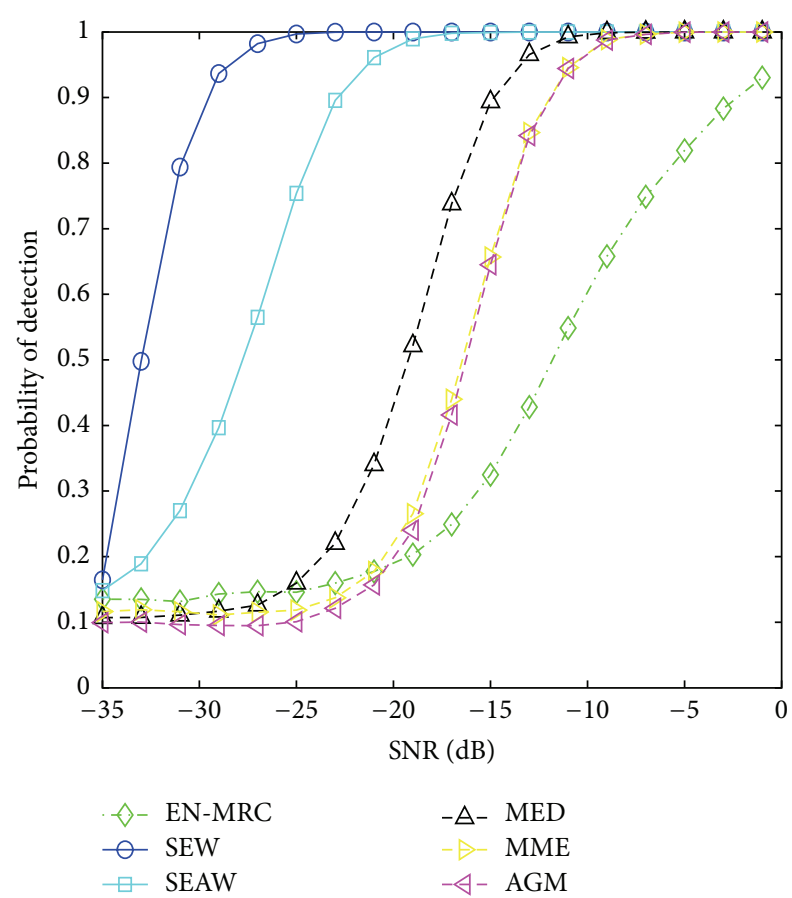

FIGURE 6: Detection performance under $N=1000$ with 2 PUs.

the replacement of energy with eigenvalue that leads to the high improvements in terms of detection probability.

In addition, as for the three new methods, we can find that SEW performs the best among these proposed methods, EN-MRC performs the worst, and the performance of SEAW is between these two methods. For example, the probability of detection of SEAW with 2 PUs (i.e., SEAW in Figure 3) is
0.5 in terms of SNR $=-15 \mathrm{~dB}$, which is in the middle of $P_{d}$ of SEW (i.e., 1) and $P_{d}$ of EN-MRC (i.e., 0.2).

According to Figures 3-6, a more interesting phenomenon can be found; that is, the SEAW's performance shifts from the lower $P_{d}$ area (close to EN-MRC) to a higher $P_{d}$ area (close to SEW) with the increasing of number of samples and number of PUs, which is like a kind of lower and upper bounds of the performance of SEAW. If we consider the performance-complexity tradeoff, the proposed SEAW can be selected as an alternative for its low complexity and relatively better performance. Hence, the SEAW may be more suitable for the application in reality.

\section{Conclusion}

This paper focuses on the problem of the eigenvalue weighting based spectrum sensing in multiantenna cognitive radio system. Through the analysis of system model, we transfer the eigenvalue weighting issue to the energy based weighting problem and derive the theoretical expression of detection threshold and probability of false alarm and finally obtain the close form expression. Considering the case of correlated signals is common in applications, we then design the signal eigenvalue based detection methods and they can achieve more higher detection probability. Simulation results verify the efficiency of the proposed algorithms.

\section{Competing Interests}

The authors declare that they have no competing interests.

\section{Acknowledgments}

This research was supported by the MSIP (Ministry of Science, ICT and Future Planning), Korea, under the ITRC (Information Technology Research Center) support program (IITP-2016-H8501-16-1019) supervised by the IITP (Institute for Information \& Communications Technology Promotion).

\section{References}

[1] Federal Communications Commission, "Notice of proposed rule making and order: facilitating opportunities for flexible, efficient, and reliable spectrum use employing cognitive radio technologies," ET Docket 03-108, Federal Communications Commission, Washington, Wash, USA, 2005.

[2] J. Mitola III and G. Q. Maguire Jr., "Cognitive radio: making software radios more personal," IEEE Personal Communications, vol. 6, no. 4, pp. 13-18, 1999.

[3] T. Yücek and H. Arslan, "A survey of spectrum sensing algorithms for cognitive radio applications," IEEE Communications Surveys and Tutorials, vol. 11, no. 1, pp. 116-130, 2009.

[4] E. Axell, G. Leus, E. G. Larsson, and H. V. Poor, "Spectrum sensing for cognitive radio: state-of-the-art and recent advances," IEEE Signal Processing Magazine, vol. 29, no. 3, pp. 101-116, 2012.

[5] M. T. Masonta, M. Mzyece, and N. Ntlatlapa, "Spectrum decision in cognitive radio networks: a survey," IEEE Communications Surveys and Tutorials, vol. 15, no. 3, pp. 1088-1107, 2013. 
[6] H. Sun, A. Nallanathan, C.-X. Wang, and Y. Chen, "Wideband spectrum sensing for cognitive radio networks: a survey," IEEE Wireless Communications, vol. 20, no. 2, pp. 74-81, 2013.

[7] X. Huang, T. Han, and N. Ansari, "On green-energy-powered cognitive radio networks," IEEE Communications Surveys and Tutorials, vol. 17, no. 2, pp. 827-842, 2015.

[8] Y. Zeng, Y.-C. Liang, A. T. Hoang, and R. Zhang, "A review on spectrum sensing for cognitive radio: challenges and solutions," EURASIP Journal on Advances in Signal Processing, vol. 2010, Article ID 381465, 15 pages, 2010.

[9] S. M. Kay, Fundamentals of Statistical Signal Processing: Detection Theory, Prentice Hall, 1998.

[10] W. A. Gardner, "Exploitation of spectral redundancy in cyclostationary signals," IEEE Signal Processing Magazine, vol. 8, no. 2, pp. 14-36, 1991.

[11] N. Han, S. H. Shon, J. O. Joo, and J. M. Kim, "Spectral correlation based signal detection method for spectrum sensing in IEEE 802.22 WRAN systems," in Proceedings of the 8th International Conference Advanced Communication Technology, pp. 17651770, Dublin, Ireland, February 2006.

[12] A. Sahai and D. Cabric, "Spectrum sensing: fundamental limits and practical challenges," in Proceedings of the IEEE International Symposium on New Frontiers in Dynamic Spectrum Access Networks (DySPAN '05), Baltimore, Md, USA, November 2005.

[13] H.-S. Chen, W. Gao, and D. G. Daut, "Signature based spectrum sensing algorithms for IEEE 802.22 WRAN," in Proceedings of the IEEE International Conference on Communications (ICC '07), pp. 6487-6492, Glasgow, UK, June 2007.

[14] H. Urkowitz, "Energy detection of unknown deterministic signals," Proceedings of the IEEE, vol. 55, no. 4, pp. 523-531, 1967.

[15] Y. Zeng, C. L. Koh, and Y.-C. Liang, "Maximum eigenvalue detection: theory and application," in Proceedings of the IEEE International Conference on Communications (ICC '08), pp. 4160-4164, Beijing, China, May 2008.

[16] R. Tandra and A. Sahai, "SNR walls for signal detection," IEEE Journal on Selected Topics in Signal Processing, vol. 2, no. 1, pp. 4-17, 2008.

[17] Y. Zeng and Y. C. Liang, "Covariance based signal detections for cognitive radio," in Proceedings of the 2nd IEEE International Symposium on New Frontiers in Dynamic Spectrum Access Networks (DySPAN '07), pp. 202-207, Dublin, Ireland, April 2007.

[18] C. Liu, M. Li, and M.-L. Jin, "Blind energy-based detection for spatial spectrum sensing," IEEE Wireless Communications Letters, vol. 4, no. 1, pp. 98-101, 2015.

[19] C. Liu and M. Jin, "Maximum-minimum spatial spectrum detection for cognitive radio using parasitic antenna arrays," in Proceedings of the IEEE/CIC International Conference on Communications in China (ICCC '14), pp. 365-369, Shanghai, China, October 2014.

[20] C. Liu, H. Li, and M. Jin, "Blind central symmetry-based feature detection for spatial spectrumsensing," IEEE Transactions on Vehicular Technology, 2016.

[21] C. Liu, S. S. Ali, R. Zhang, S.-Y. Li, J. Wang, and M.-L. Jin, “Spatial spectrum based blind spectrum sensing for multi-antenna cognitive radio system," Journal on Communications, vol. 36, no. 4, Article ID 2015087, 10 pages, 2015.

[22] Y. Zeng and Y.-C. Liang, "Eigenvalue-based spectrum sensing algorithms for cognitive radio," IEEE Transactions on Communications, vol. 57, no. 6, pp. 1784-1793, 2009.
[23] R. Zhang, T. J. Lim, Y.-C. Liang, and Y. Zeng, "Multi-antenna based spectrum sensing for cognitive radios: a GLRT approach," IEEE Transactions on Communications, vol. 58, no. 1, pp. 84-88, 2010.

[24] C. G. Tsinos and K. Berberidis, "Decentralized adaptive eigenvalue-based spectrum sensing for multiantenna cognitive radio systems," IEEE Transactions on Wireless Communications, vol. 14, no. 3, pp. 1703-1715, 2015.

[25] Z. Li, D. Wang, P. Qi, and B. Hao, "Maximum eigenvalue based sensing and power recognition for multi-antenna cognitive radio system," IEEE Transactions on Vehicular Technology, 2015.

[26] A. M. Tulino and S. Verd, Random Matrix Theory and Wireless Communivations, Now Publishers, Hanover, Mass, USA, 2004.

[27] I. M. Johnstone, "On the distribution of the largest eigenvalue in principal components analysis," The Annals of Statistics, vol. 29, no. 2, pp. 295-327, 2001.

[28] Y.-C. Liang, Y. Zeng, E. C. Y. Peh, and A. T. Hoang, "Sensingthroughput tradeoff for cognitive radio networks," IEEE Transactions on Wireless Communications, vol. 7, no. 4, pp. 1326-1337, 2008.

[29] J. Ma, G. Zhao, and Y. Li, "Soft combination and detection for cooperative spectrum sensing in cognitive radio networks," IEEE Transactions on Wireless Communications, vol. 7, no. 11, pp. 4502-4507, 2008. 

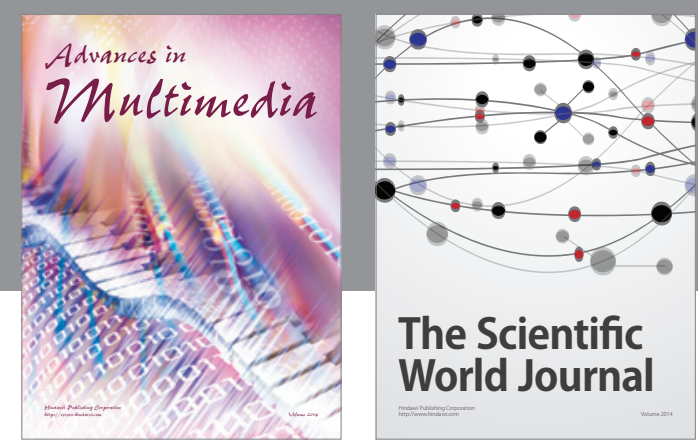

The Scientific World Journal
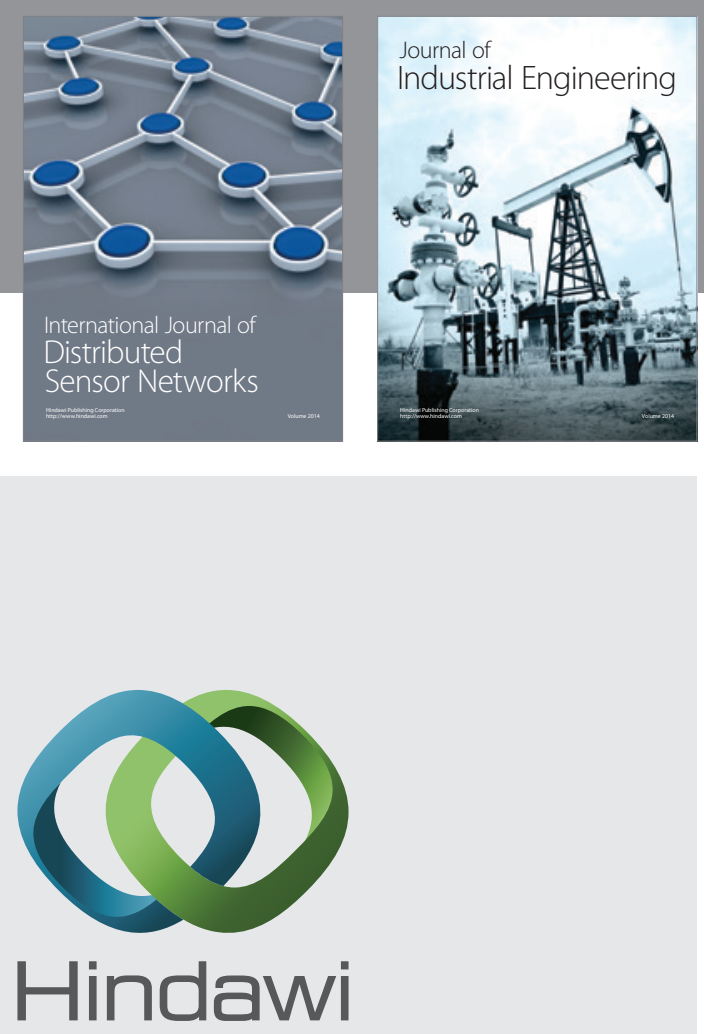

Submit your manuscripts at

http://www.hindawi.com

\section{Computer Networks} and Communications
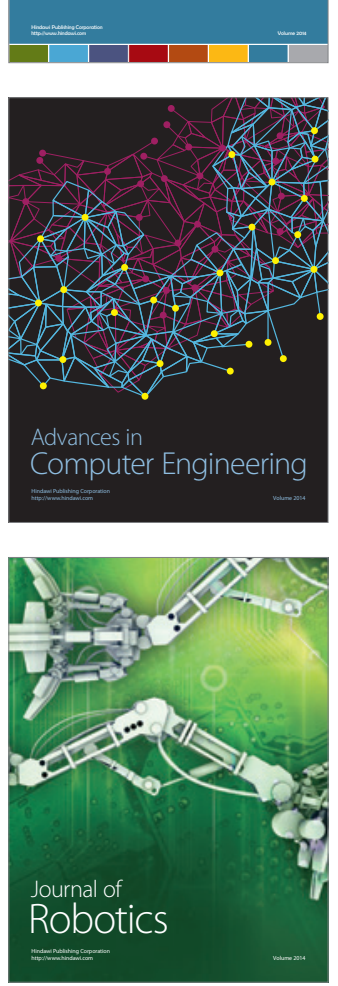
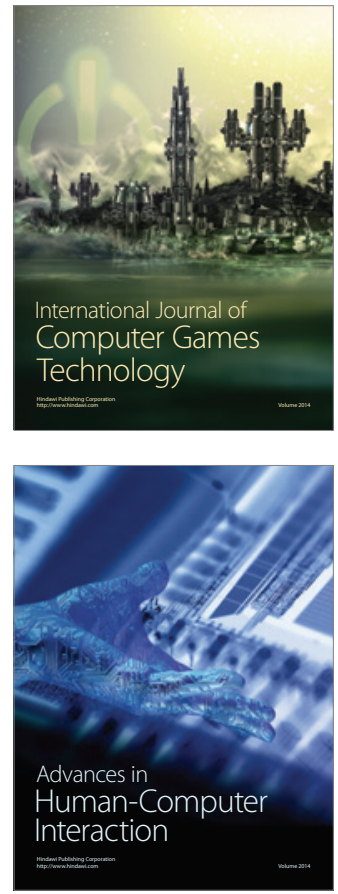
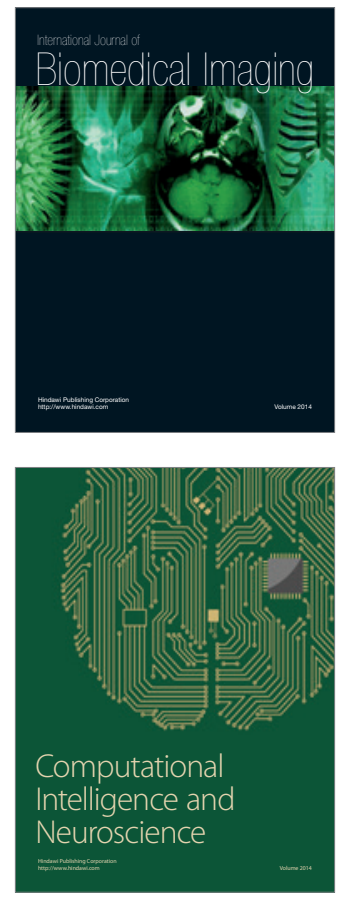
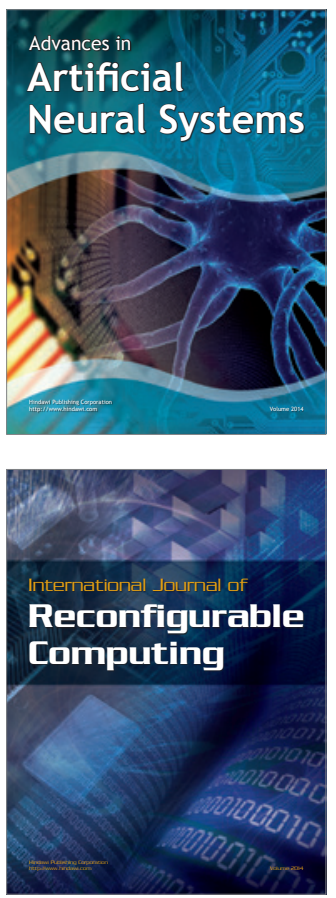
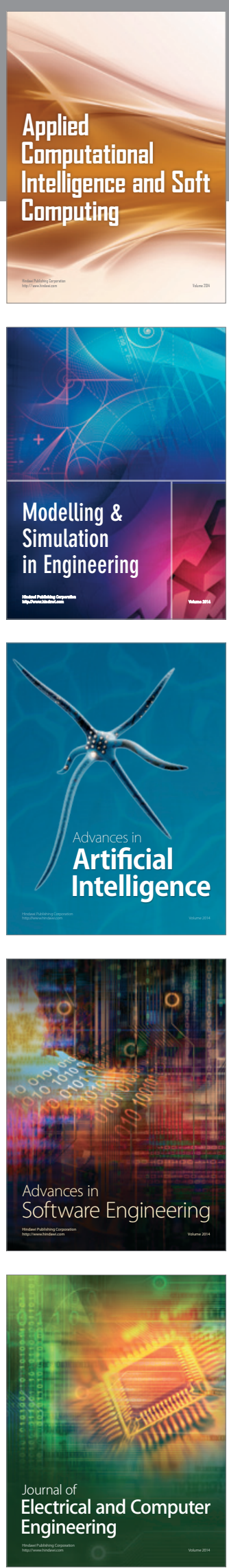